Reprod. Nutr. Dévelop. 1980, 20 (2), 443-456.

\title{
Ecdysone et reproduction chez les femelles adultes d'insectes
}

\author{
par J. HOFFMANN
}

Equipe de Recherche Associée au CNRS 118 «Biologie humorale des Insectes》 ef Laboratoire de Biologie Générale de l'Universifé Louis-Pasteur

12, rue de l'Université, 67000 Strasbourg.

Summary. Ecdysone and reproduction in adult female insects.

The data in this paper describes the involvement of ovarian ecdysone during the development of Locusta migratoria and was gathered in our laboratory during the last 4 years.

The cells of the follicular epithelium surrounding the terminal oocytes synthesize ecdysone at the end of oocyte maturation when vitellogenesis is nearly completed ; this synthesis is triggered by a folliculotropic factor probably synthesized in the medial neurosecretory cells of the brain and released by the corpora cardiaca.

Ecdysone is probably biosynthesized from cholesterol via several deoxyecdysteroids (2, 14, 22, 25-tetradeoxyecdysone ; 2, 22, 25-trideoxyecdysone, 2, 22-dideoxyecdysone ; 2-deoxyecdysone) whose presence can be ascertained by physico-chemical methods in this biological system.

Ecdysone and 2-deoxyecdysone are transferred to the oocyte (there is no quantitative data on the other deoxyecdysteroids) where 95 p. 100 accumulates as highly polar compounds presumed to be conjugated on the basis of hydrolysis experiments. If there is any, the secretion of ecdysone or ecdysone-immunoreactive molecules into the blood of the temale is only minimal.

The newly-laid egg contains practically all the ovarian ecdysteroids. The concentration of free ecdysone at that stage is $1 \mu \mathrm{M}$. As embryonic development proceeds, four distinct peaks of this concentration, reaching 4 to $8 \mu \mathrm{M}$, are observed. The first two are clearly ecdysone peaks with a low concentration of a compound presumed to be 20-hydroxyecdysone. In contrast, ecdysone peaks 3 and 4, found during postblastokinetic development, are followed by intense peaks of 20-hydroxyecdysone.

Each peak of free ecdysone concentration is correlated on an exact time-scale to the onset of cuticle deposition by the embryonic cells of the serosa or the epidermis. This leaves little doubt that, as during postembryonic development, the function of ecdysone (and/or 20 -hydroxyecdysone) in the embryo is to control cuticulogenesis.

A crucial problem is the origin of embryonic ecdysone. In the absence of biochemical data and in vitro studies on the pathway of ecdysone biosynthesis and its site in the embryo, we are reduced to working hypotheses. Before the prothoracic glands in the preblastokinetic embryo differentiate, it is probable that ecdysone peaks 1 and 2 are attained either through hydrolysis of maternal conjugates or hydroxylation of ecdysone precursors, namely 2-deoxyecdysone. It is a theoretical possibility that the differentiated prothoracic glands in the postblastokinetic embryo control the de novo ecdysone synthesis, although maternal deoxyecdysteroids or conjugates might also be used to increase the titer of free ecdysone at this stage of development. Future studies must investigate this question. 


\section{Introduction}

L'ecdysone est l'hormone qui contrôle chez les insectes, et vraisemblablement chez tous les Arthropodes, le processus de la mue. La structure de l'ecdysone est donnée par la figure 1 : il s'agit d'un stéroïde qui, contrairement aux hormones stéroïdes de Vertébrés, a gardé l'intégrité de la chaîne latérale du cholestérol, son précurseur. Elle présente d'autre part la particularité d'avoir cinq groupements hydroxyles qui la rendent nettement plus polaire que les stéroïdes hormonaux de Vertébrés.

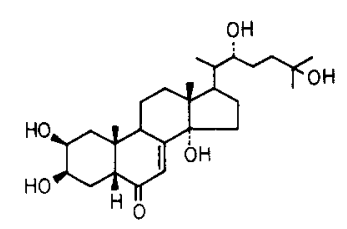

FIG. 1. - L'ecdysone ( $\alpha$-ecdysone). Cetfe hormone fut isolée par Butenandt et Karlson en 1954 à partir de $500 \mathrm{~kg}$ de pupes de Ver à soie. Sa structure fut élucidée par Karlson ef al. en 1963 et Huber et Hoppe en 1965 ( $2 \beta, 3 \beta, 14 \alpha, 22 R, 25$-pentahydroxy-5 $\beta$-cholest-7-ène-6-one).

Ecdysone ( $\alpha$-ecdysone). This hormone was isolated by Butenandt and Karlson in 1954 from $500 \mathrm{~kg}$ of silkworm pupae ; its structure $(2 \beta, 3 \beta, 14 \alpha, 22 \mathrm{R}, 25$-pentahydroxy-5 $\beta$-cholest-7-ene-6-one) was determined by Karlson ef al. (1963) and Huber and Hoppe (1965).

Ce sont les glandes prothoraciques qui sécrètent l'ecdysone ( $\alpha$-ecdysone) sous le contrôle d'une neurohormone du cerveau, l'hormone prothoracotrope (PTTH, prothoracicotropic hormone). L'ecdysone est reprise par le corps gras et les tubes de Malpighi (équivalents fonctionnels du foie et des reins des Vertébrés) dans lesquels elle subit une hydroxylation en $\mathrm{C}-20$ aboutissant à la 20-hydroxyecdysone ( $\beta$-ecdysone). La 20-hydroxyecdysone passe dans le sang qui renferme ainsi un mélange d'ecdysone et de 20-hydroxyecdysone. II n'est pour l'instant pas établi si l'hydroxylation en C-20 représente une « activation » de l'ecdysone, ou si l'ecdysone et la 20-hydroxyecdysone exercent des fonctions propres, éventuellement complémentaires.

Les insectes adultes ne présentent plus de mue ; d'autre part les glandes prothoraciques, qui synthétisent cette hormone chez les larves et les nymphes, involuent chez le jeune adulte. Ce fut donc avec surprise que furent accueillis par les endocrinologistes d'insectes les signalements de la présence d'ecdysone chez des femelles adultes de plusieurs espèces d'insectes, tels le moustique Aedes aegypti (Hagedorn et al., 1975 ; Schlaeger et al., 1974) le criquet Locusta migratoria (Hoffmann et al., 1975 ; Lagueux ef al., 1976) la reine de termite Macrotermes bellicosus (Bordereau et al., 1976 ; Delbecque et al., 1978), et la teigne des ruches Galleria mellonella (Bollenbacher ef al., 1978 ; Hsiao et Hsiao, 1979). Karlson et Stamm-Menendez avaient bien montré dès 1956 que de jeunes adultes de Bombyx mori renfermaient de l'ecdysone, mais considéraient que I'hormone, dont la concentration n'atteignait que $1 / 10$ e de celle trouvée chez la nymphe, ne représentait qu'un reliquat de l'ecdysone nymphale en voie d'être dégradée. Or les travaux que nous venons de citer montrent qu'une nette production d'ecdysone a lieu au cours de la vie adulte, au moins chez les insectes femelles.

Ces observations ont stimulé une recherche active orientée pour l'essentiel autour des axes suivants, chez les Insectes adultes: 
1. - Identification des ecdystéroïdes présents ;

2. - Biosynthèse, distribution et métabolisme de l'hormone;

3. - Fonction de l'ecdysone chez les adultes.

Ces points formeront les trois premiers chapitres de notre exposé. Nous précisons dès maintenant que chez plusieurs insectes il a été montré que l'ecdysone présente chez la femelle adulte était transférée à l'ovocyte et retrouvée dans les œufs au début du développement embryonnaire. Le 4 e chapitre de notre étude sera consacrée pour cette raison à l'ecdysone chez les embryons.

\section{Résultats et discussions}

A. - Identification des ecdystéroïdes présents chez les insectes adultes.

Les premiers travaux rapportant la présence d'ecdysone chez des femelles adultes d'insectes au cours de la reproduction étaient basés sur l'emploi de dosages biolo-

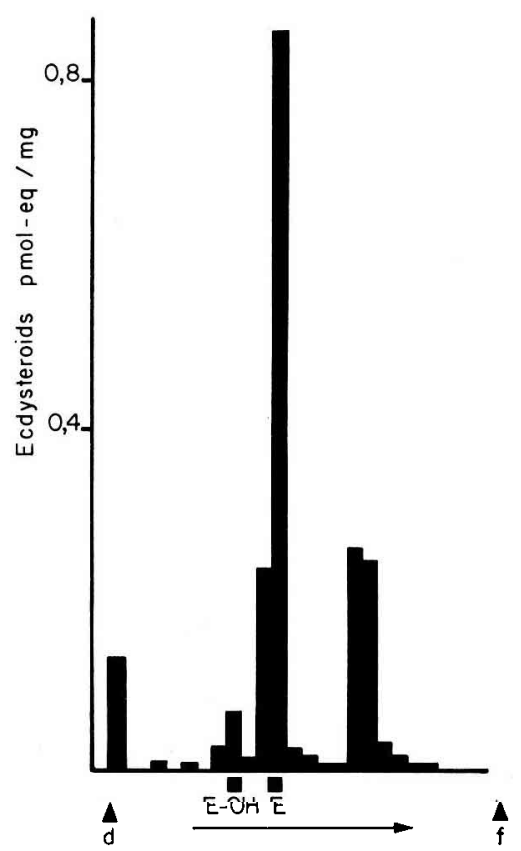

FIG. 2. - Purification d'extraits ovariens de femelles adultes de Locusta migratoria en chromatographie sur couche mince (plaques de silice 60,0,25 mm, HF 254, Merck ; chloroforme-méthanol : $80 \mathrm{vol} / 20$ vol ; double migration) suivie d'élution par bandes de $5 \mathrm{~mm}$ (triple élution à l'éthanol 950) et dosage radioimmunologique (anticorps anti-succinyl-20-hydroxyecdysone-HSA dilué $1 / 10000$; de Reggi ef al., 1975 ; antigène : 23-24 ${ }^{3} \mathrm{H}$-ecdysone, $60 \mathrm{Ci} / \mathrm{mmol}$ ). d, dépôt ef $\mathrm{f}$, front de migration ; $\mathrm{E}$ ef $\mathrm{E}-\mathrm{OH}$, migration d'ecdysone et de 20-hydroxyecdysone de référence (SIMES, Milan).

Purification of ovarian extracts of adult female Locusta migratoria by thin-layer chromatography (silica gel plates $60,0.25 \mathrm{~mm}$, HF 254, Merck ; chloroform-methanol : $80 \mathrm{vol} / 20 \mathrm{vol}$; double migration) followed by $5 \mathrm{~mm}$ band elution (triple elution with $95^{\circ}$ éthanol) and radioimmunological assay (RIA) (1/10 000 dilution of antibody anti-succinyl-20-hydroxyecdysone-HSA, de Reggi et al., 1975 ; antigen : $23-24{ }^{3} \mathrm{H}$-ecdysone, $\left.60 \mathrm{Ci} / \mathrm{mmol}\right)$. $\mathrm{d}$ : deposit ; $\mathrm{f}$ : migration front ; $\mathrm{E}$ : ecdysone migration ; $\mathrm{E}-\mathrm{OH}$ : migration of reference 20-hydroxyecdysone (SIMES, Milan). 
giques ou radioimmunologiques. Des recherches furent entreprises pour montrer l'identité physico-chimique entre le produit trouvé chez les insectes adultes et l'ecdysone présente au cours du développement larvaire et nymphal : dès 1976, cette identité fut démontrée par spectrométrie de masse, dans des extraits ovariens de Locusta migratoria (Luu Bang et al., 1976).

II s'est avéré rapidement que les ovaires adultes renfermaient d'autres molécules de la famille des ecdystéroïdes. La figure 2 montre à titre d'exemple que dans les extraits d'ovaires de Locusta plusieurs composés présentent une réaction de croisement avec un anticorps anti-20-hydroxyecdysone (obtenu par injection d'un dérivé succinyl20-hydroxyecdysone-HSA ; voir De Reggi ef al., 1975). Dans des extraits de plusieurs milliers d'ovaires de Locusta migratoria, notre groupe (Hetru et al., 1978) a décrit 4 ecdystéroïdes de polarités intermédiaires entre celle de l'ecdysone et celle du cholestérol. L'analyse par chromatographie en phase gazeuse couplée à la spectrométrie de masse de ces produits (A, B, C et D) a permis de proposer les structures suivantes :
$A: 2$ - deoxyecdysone,
B : 2,22-bis-deoxyecdysone,
C : 2,22,25-tri-deoxyecdysone,
D : 2,14,22,25-tétra-deoxyecdysone.

Cette interprétation vient d'être confirmée pour les produits $A, C$ et $D$ par le traitement de produits de référence dans les mêmes conditions analytiques (Hetru, non publié).

La figure 3 qui présente les produits A à D dans l'ordre de leur polarité croissante, entre le cholestérol et l'ecdysone, apparaît comme un schéma de biosynthèse possible de l'ecdysone.
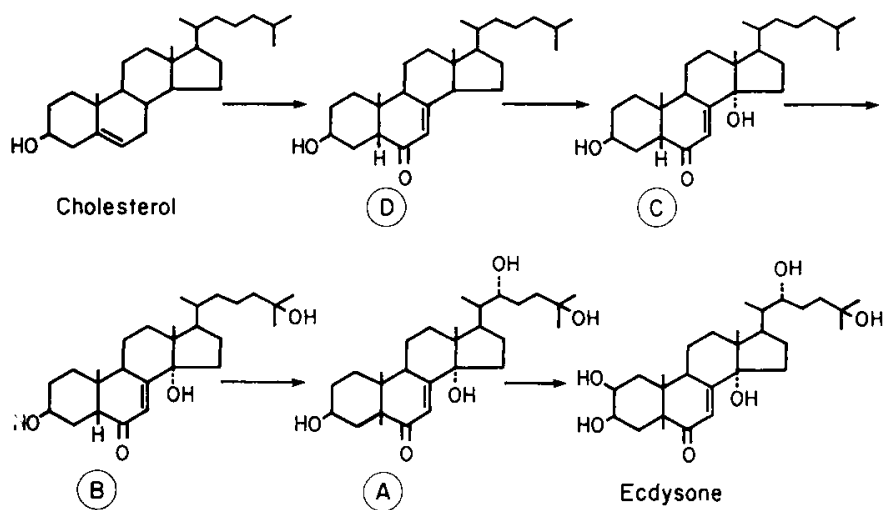

FIG. 3. - - Voie probable de la biosynthèse de l'ecdysone dans les ovaires de Locusta migratoria. Les six produits de la figure 3 sont présents dans les ovaires au moment de la biosynthèse d'ecdysone. Aucun autre ecdystéroïde de polarité intermédiaire entre le cholestérol et l'ecdysone n'est présent dans les ovaires. Détails dans Hetru et al. (1978).

Probable pathway of ecdysone biosynthesis in Locusta migratoria ovaries. The six products of figure 3 are present in the ovaries at ecdysone biosynthesis. No other ecdysteroid, of polarity intermediate befween cholesterol and ecdysone, is present in the ovaries (see details in Hetru et al., 1978). 
Les molécules immunoréactives moins polaires que l'ecdysone représentées sur la figure 2 correspondent à la 2-déoxyecdysone ; les produits $C$ et $D$ ne présentent pas de réactions de croisement avec l'ecdysone pour l'anticorps.

Sur la même figure, nous voyons 2 pics d'immunoréactivité dans la zone plus polaire que l'ecdysone : l'un co-migre avec la 20-hydroxyecdysone et a le même temps de rétention en chromatographie liquide à haute performance que la substance de référence. L'autre pic représente un mélange de produits très polaires, qui ne migrent pratiquement pas dans le système de solvants utilisés ; soumis à hydrolyse acide en présence de jus commercial de Hélix pomatia, ces produits libèrent de l'ecdysone et de la 2-déoxyecdysone. La quantification au RIA de l'ecdysone libérée par hydrolyse permet de calculer que, dans un ovaire de Locusta en fin de maturation, la concentration en ecdysone conjuguée dépasse très largement la concentration en ecdysone libre.

\section{B. - Biosynthèse ef distribution de l'ecdysone chez les insectes adultes.}

En 1975, Hagedorn et collaborateurs ont montré que des ovaires explantés de femelles adultes d'Aedes aegypti sécrétaient dans le milieu d'incubation de l'ecdysone (dosage radioimmunologique après purification en chromatographie en phase liquide sous haute pression). Comme ces auteurs avaient omis de doser l'ecdysone dans les

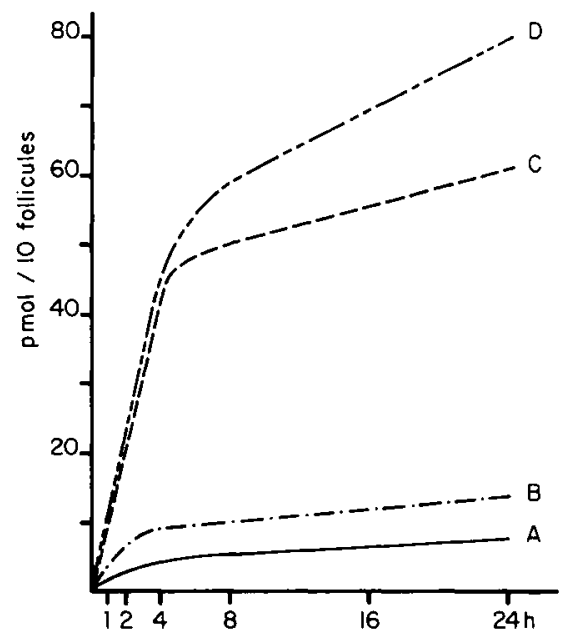

FIG. 4. - Cinétique de sécrétion d'ecdysone par les cellules folliculaires d'ovaires de Locusta migratoria en fin de mafuration. Le degré d'avancement de l'ovogenèse est estimé par mesure des longueurs des ovocyłes terminaux (début de vifellogenèse : $1,2 \mathrm{~mm}$; ponte ovarienne : $6,5 \mathrm{~mm}$ ). Les follicules étaient prélevés sur des femelles aux stades suivants : (A) longueur des ovocytes terminaux: $4,3 \mathrm{~mm}$; (B) : $5,3 \mathrm{~mm}$; (C) : $5,5 \mathrm{~mm}$; (D) : 5,7 $\mathrm{mm}$ ). Abscisses : durée de l'incubation, en heures ; ordonnées : quantité d'ecdysone sécrétée en pmol-équivalents par 10 follicules. Déterminations radioimmunologiques d'extraits éthyliques. Défails dans Goltzené et al., 1978.

Kinetics of follicle cell ecdysone secretion in Locusta migratoria ovaries at the end of maturation. The stage of oogenesis is estimated by measuring terminal oocyte length (onset of vitellogenesis : $1.2 \mathrm{~mm}$; at egg laying: $6.5 \mathrm{~mm}$ ). The follicles were sampled at the following terminal oocyte lengths: (A) $4.3 \mathrm{~mm}$, (B) $5.3 \mathrm{~mm}$, (C) $5.5 \mathrm{~mm}$, (D) $5.7 \mathrm{~mm}$. Abscissas : length of incubation (in hrs) :Ordinates : amount of ecdysone secretion (in pmol-equivalents) per 10 follicles. RIA of ethylic extracts (see details in Goltzene ef al., 1978). 
ovaires avant et après incubation, il n'était pas évident que la sécrétion in vitro correspondait à de l'hormone nouvellement synthétisée dans les ovaires. En 1977, notre groupe a pu montrer chez Locusta migratoria que des ovaires entiers de femelles en fin de maturation ovarienne synthétisaient effectivement de l'ecdysone in vitro ; après dissection des ovaires et incubations séparées des ovocytes, des cellules de l'épithélium folliculaire, des glandes accessoires et des oviductes, nous avons pu préciser que le site de biosynthèse dans l'ovaire éfait l'épithélium folliculaire entourant les ovocytes terminaux (Lagueux et al., 1977). Nous avons repris ces études récemment, toujours chez Locusta, et montré que l'intensité de l'activité ecdysiosynthétique des cellules de l'épithélium folliculaire dépend strictement du stade de maturation de l'ovaire sur lequel ces cellules sont prélevées (Goltzené ef al., 1978). La figure 4 illustre quelques-unes de ces observations. Ces résultats ont montré par ailleurs qu'à l'intérieur d'un cycle de maturation ovarienne de Locusta, la période d'activité de synthèse d'ecdysone dans les cellules folliculaires était relativement courte $(8$ à $12 \mathrm{~h}$ pour une durée de cycle de plusieurs jours) et qu'elle se situait vers la fin de la maturation des ovocytes, quand la vitellogenèse touchait pratiquement à sa fin.

Plusieurs arguments militent actuellement en faveur de l'existence d'un contrôle neurohormonal de la biosynthèse d'ecdysone chez les femelles adultes; en effet une série d'expériences réalisées chez Locusta (fig. 5 et 6) montrent que la biosynthèse d'ecdysone dans les ovaires n'a pas lieu si les centres neurosécréteurs du cerveau (pars intercerebralis) sont électrocoagulés ou si les corpora cardiaca (organe neurohémal présentant lui-même un lobe glandulaire) sont extirpés. A l'opposé, l'implantation de centres neurosécréteurs cérébraux ou de corpora cardiaca supplémentaires avance notablement la biosynthèse d'ecdysone chez des femelles adultes normales, à une condition : il faut que les donneurs de complexes cerveau-corpora cardiaca soient euxmêmes des femelles adultes; l'implantation de complexes provenant de larves ou d'adultes de sexe mâle est sans effet. Il est ainsi très vraisemblable que la biosynthèse de l'ecdysone dans les cellules folliculaires est régulée par un mécanisme analogue à celui qui contrôle la production d'ecdysone dans les glandes prothoraciques au cours du développement postembryonnaire. Des résultats qui se rapprochent des nôtres ont été obtenus récemment chez Aedes aegypti (Hagedorn, communication personnelle).

L'ecdysone synthétisée dans les cellules folliculaires passe dans les ovocyłes où elle s'accumule. La figure 7 montre l'évolution du taux d'hormone observé dans les ovaires de Locusta à la fin de deux cycles successifs de maturation : le taux augmente très rapidement lorsque les ovocytes terminaux atteignent une longueur de 5,5 à $6 \mathrm{~mm}$, période vers laquelle l'activité ecdysiosynthétique des cellules folliculaires atteint son maximum (voir fig. 4). Au moment de la ponte des ovocytes terminaux, le taux d'ecdysone de l'ovaire tombe à des valeurs insignifiantes, alors que dans les œufs fraîchement pondus, l'hormone est intégralement retrouvée.

Ce « transfert » de l'ecdysone de la femelle adulte à l'œuf est observé chez d'autres insectes, comme la reine de termite (Delbecque et al., 1978), le ver à soie (Ohnishi ef al., $1977 b$ ) et la teigne de la ruche de l'abeille (Bollenbacher et al., 1978 ; Hsiao et Hsiao, 1979). Il n'est cependant pas absolument certain que ce soit le cas chez tous les insectes.

Une sécrétion d'ecdysone à partir des ovaires vers le sang de la femelle adulte n'a pas pu être mise en évidence avec certitude chez Locusta (il faut rappeler que des ovo- 

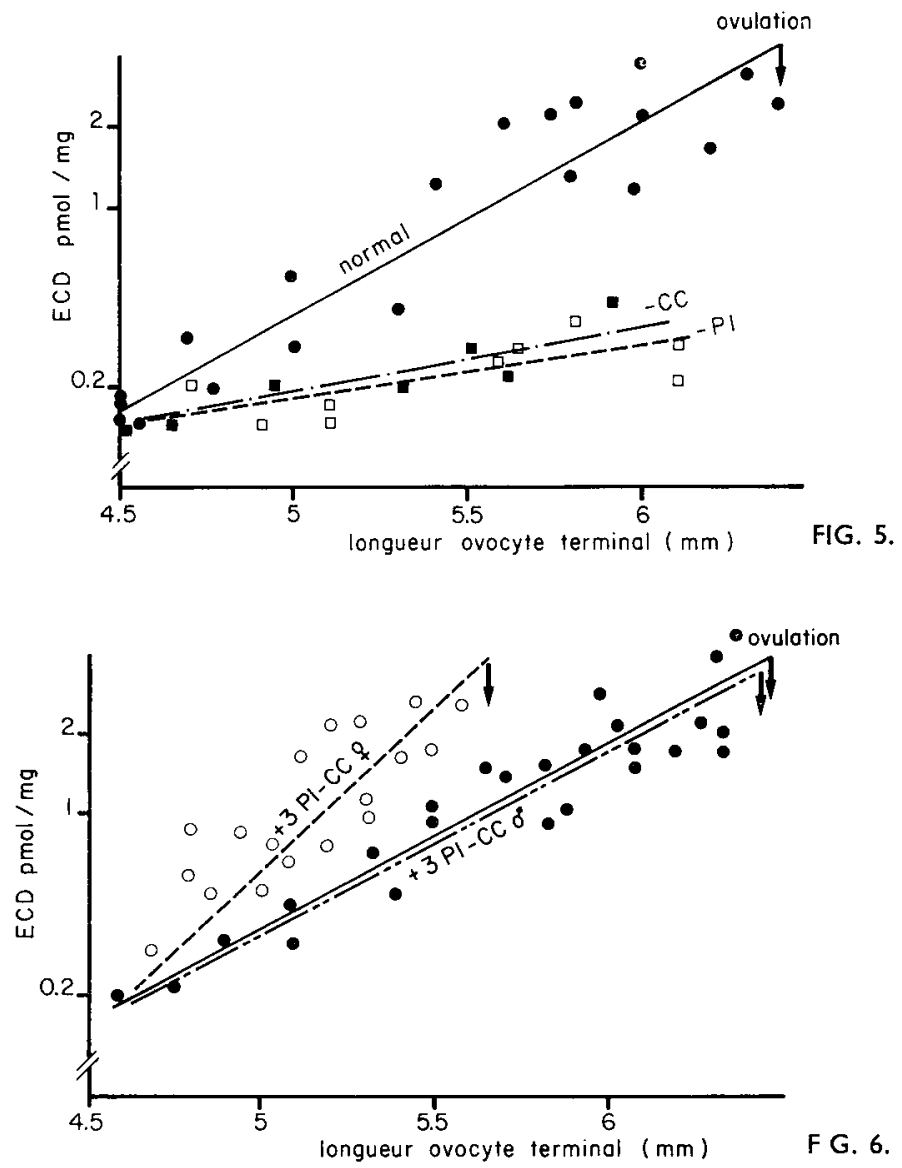

FiG. 5 et 6. - Recherches sur un contrôle neuro-hormonal de la production d'ecdysone dans les ovaires de Locusta migratoria. Abscisses : état du développement de l'ovaire exprimé en fonction de la longueur des ovocytes terminaux (ponte vers $6,5 \mathrm{~mm}$ ) ; ordonnées : concentration ovarienne en ecdystéroïdes, en pmol-équivalents par $\mathrm{mg}$ d'ovaire (dosages radioimmunologiques d'extraits éthyliques d'ovaires entiers).

Study of neuro-hormonal control of ecdysone production in Locusta migratoria ovaries. Abscissas : state of ovarian development in relation to terminal oocyte length (Laying at $6.5 \mathrm{~mm}$ ) ; Ordinate : ovarian ecdysteroid concentration (in pmol equivalents) per mg of ovary (RIA of ethylic extracts of whole ovaries).

L'évolution de la concentration en ecdystéroïdes est donnée en fin de maturation dans les ovaires normaux (points noirs) et chez les animaux expérimentaux suivants : femelles adultes après électrocoagulation du cerveau (stade de l'opération : $4 \mathrm{~mm}$ de longueur ovocytaire) ou ablation des corpora cardiaca (même stade) et femelles adultes ayant reçu l'implantation de 3 complexes cerveau-corpora cardiaca supplémentaires prélevés sur des donneurs femelles adultes (P) ou mâles adultes (3) (stade de l'opération : $2 \mathrm{~mm}$ de longueur ovocytaire).

The change in ecdysone concentration is shown at the end of normal ovarian maturation (black points) in the following experimental animals : adult females with brain electrocoagulation (operative stage : $4 \mathrm{~mm}$ oocyte length) or excision of the corpora cardiaca (same stage) and adult females implanted with 3 extra brain-corpora cardiaca systems taken from adult female (i) or male ( $\left.\delta^{*}\right)$ donors (operative stage : $2 \mathrm{~mm}$ oocyte length). 
cyłes terminaux en fin de maturation sont résorbés régulièrement, ce qui pourrait expliquer la présence occasionnelle de molécules (ecdysone-) immunoréactives dans le sang ou le corps gras de certaines femelles adultes). Une sécrétion d'ecdysone dans le sang est postulée par contre chez le moustique Aedes aegypti ; d'après les données de Bollenbacher et al. (1978), chez Galleria mellonella, 30 p. 100 de l'ecdysone ovarienne passeraient dans le sang chez cette espèce et 70 p. 100 seraient transférés à l'œuf.

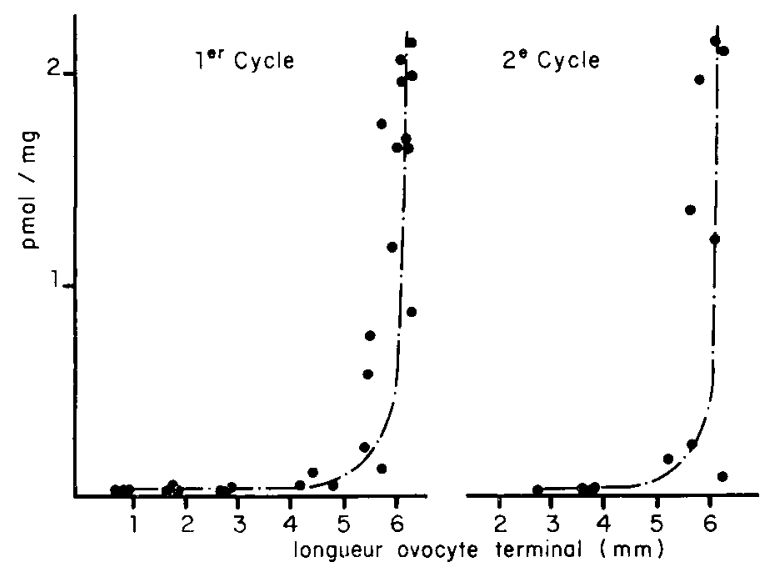

FIG. 7. - Evolution du faux d'ecdysone au cours de deux cycles successifs de maturation ovocyfaire. Abscisses: développement ovocytaire, exprimé en $\mathrm{mm}$ de longueur des ovocytes terminaux (ponte vers $6,5 \mathrm{~m}$ ); ordonnées : concentration en ecdysone, exprimée en pmol-équivalents par $\mathrm{mg}$ d'ovaire. Dosages radioimmunologiques d'ecdysone purifiée par chromatographie sur couche mince à partir d'extraits éthyliques d'ovaires (mêmes techniques que pour fig. 2).

Change in ecdysone level during two successive cycles of oocyte maturation. Abscissas : oocyte development (in $\mathrm{mm}$ of terminal oocyte length ; laying at $6.5 \mathrm{~mm}$ ) ; Ordinates : ecdysone concentration (in pmol-equivalents) per $\mathrm{mg}$ of ovary. RIA of ecdysone purified by thinlayer chromatography from ovarian ethylic extracts (same techniques as fig. 2).

A l'intérieur de l'ovocyte de Locusta migratoria, l'ecdysone est liée pour plus de 90 p. 100 à une macromolécule. La caractérisation de cette liaison est en cours chez Locusta. Mais la fixation de l'ecdysone à des macromolécules (protéiques par exemple) ne présente qu'un des volets du problème du transfert de l'hormone des cellules folliculaires à l'œuf. Comme nous l'avons déjà vu, une importante fraction de l'ecdysone (eł de la 2-déoxyecdysone) est présente sous forme de conjugués dans l'ovoplasme (conjugués aisément hydrolysables par le jus commercial d'Helix pomatia). Ces conjugués sont formés dans l'ovaire même. Nous reviendrons plus loin sur la signification possible de cette conjugaison. Précisons que des conjugués d'ecdysone sont également décrits dans les ovaires et les œufs fraîchement pondus de Schistocerca gregaria (Gande et Morgan, 1979), de Galleria mellonella (Hsiao et Hsiao, 1979) et Bombyx mori (Ohnishi et al., 1977a).

\section{C. - Fonction de l'ecdysone ovarienne.}

La découverte de la présence d'ecdysone dans les ovaires en maturation chez des femelles de plusieurs espèces d'insectes a évidemment soulevé le problème de la fonction de cette hormone à une période de la vie où les insectes ne muent pas. 
C'est chez le moustique qu'une fonction précise fut proposée en premier lieu : le groupe de Hagedorn (Fallon et al., 1974 ; Hagedorn, 1974 ; Hagedorn et al., 1975) a décrit la stimulation de la synthèse de vitellogénine au niveau du corps gras d'adultes femelles de moustique sous l'influence d'ecdysone exogène. Ces auteurs ont conclu que l'ecdysone synthétisée par les ovaires était sécrétée dans le sang et agissait sur le corps gras (éventuellement après avoir été hydroxylée en 20-hydroxyecdysone) où elle stimulait la synthèse de vitellogénine. Ces travaux, qui ont initialement reçu une très large audience, appellent cependant certaines réserves : 1) les auteurs n'ont pas pu démontrer que les ovaires sécrètent effectivement de l'ecdysone dans le sang dans les conditions physiologiques; 2) les doses nécessaires pour stimuler expérimentalement in vivo et in vitro la production de vitellogénine dans le corps gras sont 10000 fois supérieures aux doses physiologiques ; une dose qui ne dépasse que de quelques centaines de fois la dose physiologique est incapable de maintenir la synthèse de vitellogénine dans le corps gras de femelles adultes ovariectomisées (Borovsky et Van Handel, 1979) ; 3) la transplantation d'ovaires (source d'hormone) dans des femelles adultes non vitellogéniques ne stimule pas la synthèse de vitellogénine (Borovsky ef Van Handel, 1979) ; 4) les auteurs n'ont pas pu démontrer que la stimulation de la synthèse protéique dans le corps gras est spécifique pour la vitellogénine : en fait, l'injection d'ecdysome stimule de façon non spécifique la synthèse de toutes les protéines du corps gras chez Bombyx mori adultes mâles et femelles (Ono ef al., 1975) et Locusta migratoria (Emmerich ef Hoffmann, observations non publiées).

II est certainement prudent de dire pour l'instant que l'hypothèse proposée par Hagedorn et collaborateurs pour la fonction de l'ecdysone chez la femelle adulte du moustique n'est pas démontrée. Le fût-elle, qu'il faudrait considérer le cas du moustique comme un cas particulier, dans la mesure où chez les autres insectes que nous avons cités jusqu'ici, la stimulation de la synthèse de vitellogénine est réalisée par l'hormone juvénile et elle est nettement antérieure à la synthèse d'ecdysone dans l'ovaire (ceci est particulièrement bien éfabli chez Locusta, voir Lagueux ef al., 1977 et Goltzené, 1977). D'autre part, comme nous l'avons déjà signalé, l'ecdysone n'est pas présente dans le sang et dans les tissus périphériques dans la majorité des cas que nous avons cités.

Plusieurs autres hypothèses ont été envisagées pour la fonction de l'ecdysone chez la femelle adulte, mais aucune pour l'instant n'est démontrée. De sorte qu'il est légitime de se demander si l'ecdysone a réellement un rôle à jouer chez la femelle, ou si sa fonction n'est pas à rechercher chez l'embryon. Comme nous l'avons vu, chez la plupart des insectes étudiés sous ce rapport, l'hormone présente dans les ovaires est pratiquement entièrement retrouvée dans les œufs fraîchement pondus. Nous examinerons la destinée de cette hormone au cours du développement embryonnaire dans le prochain chapitre.

\section{D. - L'ecdysone chez l'embryon.}

L'œuf fraîchement pondu de Locusta renferme des concentrations considérables d'ecdysone. La concentration totale (fraction conjuguée et fraction libre) de cette hormone dans l'œuf est voisine de $100 \mu \mathrm{M}$. Il est important de souligner que seuls 2 à 3 p. 100 de l'ecdysone sont présents sous forme réellement « libre », le reste étant soit présent 
sous forme de conjugués, soit lié à des macromolécules. Le transfert d'ecdystéroïdes ne se limite pas à la seule ecdysone : la 2-déoxyecdysone (et vraisemblablement la 2,22-bis-déoxyecdysone ef la 2,22,25-tri-déoxyecdysone est également trouvée en grandes concentrations dans l'œuf fraîchement pondu (environ 30 à 40 p. 100 de la concentration de l'ecdysone chez Locusta ; concentration voisine de celle de l'ecdysone chez Bombyx, Ohnishi ef al., 1977a) ; la 2-déoxy-ecdysone est d'ailleurs également présente en tant que conjugué ; une partie est liée d̀ des macromolécules.

Le problème du rôle des ecdystéroïdes d'origine maternelle chez l'embryon se pose de façon complexe ; pour l'instant il n'a pas été possible de supprimer expérimen-

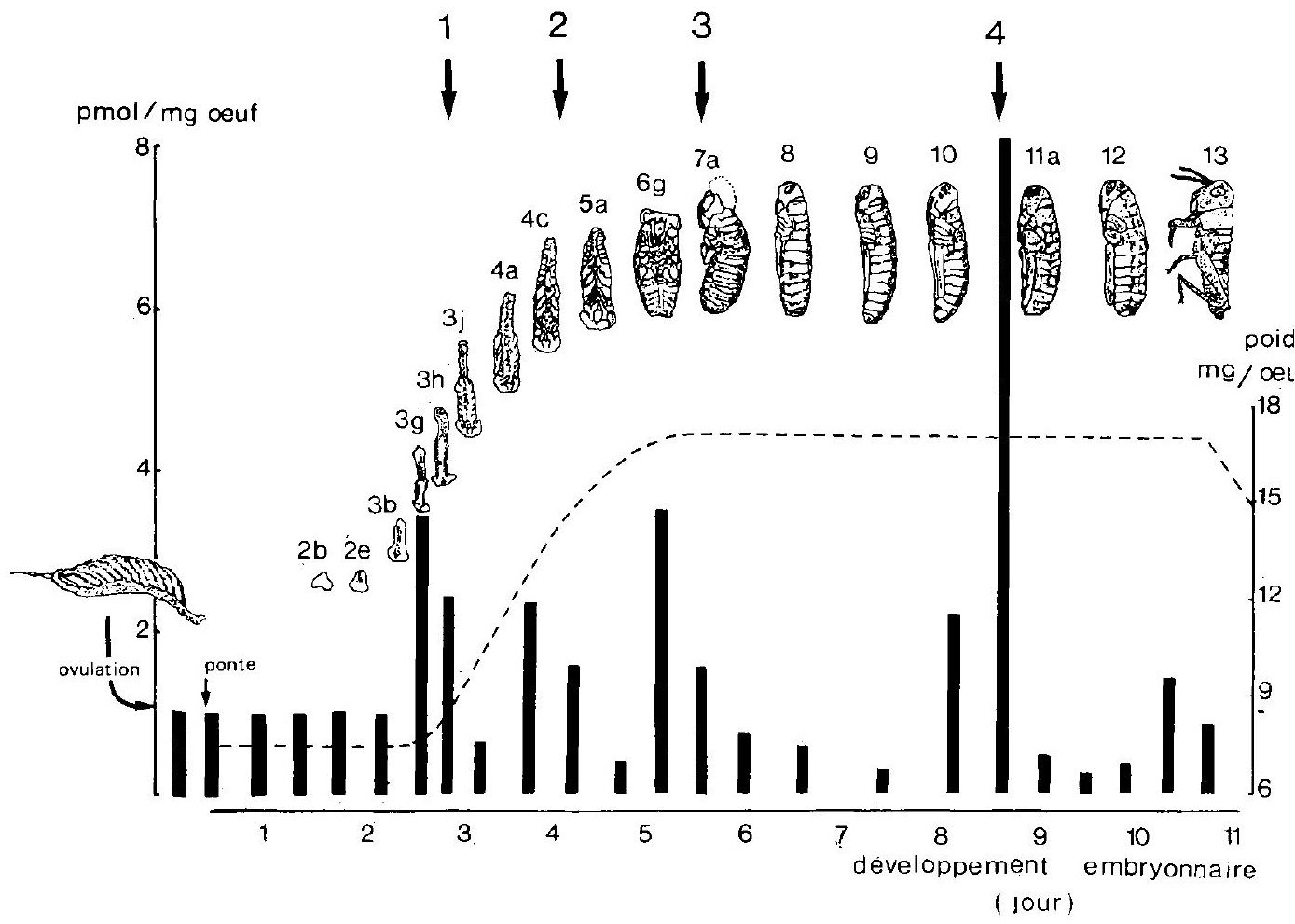

FIG. 8. - Titre d'ecdysone libre en relation avec le développement embryonnaire de Locusta migratoria. L'axe des abscisses présente la durée du développement embryonnaire, en jours (11 jours en moyenne de la ponte à l'éclosion) ; les stades de développement de l'embryon sont figurés par les dessins $2 b$ à 13 dans la figure. L'axe des ordonnées représente le taux d'ecdysone dans les œufs (dosages radioimmunologiques d'extraits éthyliques d'œufs après purification en chromatographie en couche mince et élution de l'ecdysone seule); moyennes de trois séries indépendantes de déterminations sur des lots de 30 œufs par série. Les flèches 1 à 4 indiquent les stades des 4 dépôts cuticulaires dans l'œuf au cours du développement.

Titer of free ecdysone in relation to embryonic development of Locusta migratoria. Abscissas : time-course of embryonic development (in days ; mean 11 days from laying to hatching) ; embryonic stages of development are shown in drawings $2 b$ to 13 of the figure; Ordinates : ecdysone level in eggs (RIA of egg ethylic extracts after purification by thin-layer chromatography and elution of ecdysone alone) ; means of three different assay series of lots of 30 eggs per series. Arrows 1 to 4 show the stages of the four cuticle deposits in the egg during development. 
talement le transfert, mais des efforts sont entrepris dans ce sens. Dans l'immédiat, nous avons suivi chez Locusta l'évolution du taux des différents ecdystéroïdes de la ponte à l'éclosion, et essayé d'établir des corrélations aussi précises que possible entre les principales étapes morphogénétiques chez l'embryon et les fluctuations des taux hormonaux.

La concentration d'ecdysone non conjuguée qui est de $1 \mu \mathrm{M}$ dans l'œuf au moment de la ponte, présente 4 pics au cours du développement embryonnaire (valeurs des pics : 3 d̀ $8 \mu \mathrm{M}$, voir fig. 8). En faisant en parallèle aux dosages une étude des dépôts de cuticules dans l'œuf, nous avons pu montrer que chaque pic d'ecdysone est concomitant (ou précède de quelques heures) de l'apparition d'une cuticule. Le premier pic hormonal est concomitant de la synthèse d'une cuticule par les cellules sérosales. Les trois autres pics sont concomitants des dépôts successifs des trois cuticules de l'épiderme embryonnaire. A chaque nouveau dépôt, l'ancienne cuticule se détache de l'épiderme (apolyse) ; la troisième cuticule qui est sécrétée par l'épiderme embryonnaire sert de cuticule à la larve au moment de l'éclosion (1 re cuticule larvaire).

Ces résultats laissent peu de doute que l'une au moins des fonctions de l'ecdysone chez l'embryon est le contrôle du dépôt des cuticules. Telle est, rappelons-le, une fonction essentielle de l'ecdysone au cours du développement postembryonnaire (larves et nymphes).

Le point critique est de connaître le rapport exact entre les pics d'ecdysone observés chez l'embryon et les ecdystéroïdes d'origine maternelle. Il est hors de doute que l'embryon «produit » des quantités importantes d'ecdysone à 4 reprises pendant son développement. Est-ce que cette «production » traduit : 1) une néosynthèse d'ecdysone à partir de cholestérol ; 2) une hydrolyse des conjugués d'origine maternelle ; 3) une hydroxylation de la 2-déoxyecdysone d'origine maternelle (ou deux hydroxylations de la 2,22-bis-déoxyecdysone également d'origine maternelle) ? Nous ne possédons pas la réponse à ces questions pour l'instant. II est d'ailleurs possible que la réponse soit différente quant à la «production » de l'ecdysone au moment des pics précoces ef des pics tardifs : les glandes prothoraciques se différencient dans l'embryon de Locusta après le pic d'ecdysone 2, et on peut admettre à titre d'hypothèse, qu'elles soient d'emblée capables de convertir le cholestérol en ecdysone, comme elles le feront pendant la vie larvaire. Effectivement, chez Locusta, Jacquot (1979) vient de montrer que le fragment tête-prothorax d'un embryon explanté au moment du pic 3 (stade 6, fig. 8) continue in vitro à produire de l'ecdysone. A notre surprise cependant, il en est de même pour les fragments méso- ef méta-thoraciques et pour l'abdomen isolé (Jacquot, 1979). Ce résultat, même s'il ne permet pas de trancher le problème de la biosynthèse de l'ecdysone, pourrait expliquer les observations de plusieurs embryologistes, qui avaient nofé que des abdomens d'embryons d'insectes continuaient à muer in vitro en l'absence des glandes prothoraciques embryonnaires (Haget, 1953 ; Müller, 1963 ; Sbrenna-Micciarelli ef Sbrenna, 1972).

\section{Conclusion}

Il y a à peine trois ans que la preuve irréfułable a été apportée que les ovaires de femelles adultes d'insectes contiennent effectivement de fortes concentrations de l'hormone qui contrôle la mue au cours du développement larvaire et nymphal. II n'est pas 
étonnant que nous soyons encore à l'heure actuelle en présence de plus de questions que de réponses en ce qui concerne ce problème nouveau de l'endocrinologie des insectes. Quoique fragmentaires, les résultats disponibles permettent néanmoins de tirer certaines conclusions.

Arrivé à un certain stade de maturation, l'ovaire d'insectes femelles synthétise de l'ecdysone. Chez la plupart des insectes, ce stade de maturation n'est réalisé que chez l'adulte, mais chez le ver à soie, par exemple, où la maturation ovarienne est bien avancée avant la mue en adulte, l'ovaire synthétise de l'ecdysone dès le stade nymphal (Legay ef al., 1976 ; Ohnishi et al., 1977b).

Il est vraisemblable que la synthèse démarre sous l'influence d'un stimulus neurohormonal, ef ceci à un stade où la vitellogenèse est bien avancée (exception faite de Aedes peut-être). La biosynthèse de l'ecdysone se fait à partir du cholestérol ; l'apparition d'une double liaison en $\Delta 7$, l'introduction d'une cétone en C-6, la réduction de $\Delta 5$ avec introduction d'un $\mathrm{H}$ en $5 \beta$ (noyaux $\mathrm{A} / \mathrm{B}$ en cis), précèdent les hydroxylations en $C-14-\alpha, C-25$, et C-22. L'hydroxylation en C-2 apparaît comme la dernière étape de la biosynthèse ; il est significatif qu'elle ne soit pas d'emblée réalisée sur toutes les molécules entrant dans la voie de biosynthèse, comme les taux de 2-déoxyecdysone sont importants dans l'ovaire.

La biosynthèse de l'ecdysone a lieu dans les cellules de l'épithélium folliculaire. L'ecdysone ef la 2-déoxyecdysone entrent dans l'ovocyte et y sont accumulés en grande partie sous forme de conjugués.

II n'existe pas actuellement de preuve pour une sécrétion massive de l'ecdysone ovarienne dans le sang ni pour une fonction de l'hormone dans un quelconque tissu périphérique de la femelle adulte. Les recherches dans ce sens doivent être poursuivies. Par contre il est établi chez plusieurs insectes que l'hormone synthétisée dans l'ovaire est retrouvée pratiquement intégralement dans l'œuf fraîchement pondu (il en est de même pour la 2-déoxyecdysone).

Au cours du développement embryonnaire, 4 cuticules successives sont synthétisées dans l'œuf ; chaque dépôt cuticulaire est concomitant d'un pic d'ecdysone libre. Les deux premiers pics sont observés avant que les glandes endocrines embryonnaires ne soient différenciées. Les résultats disponibles pour l'instant sont compatibles avec l'hypothèse que l'hormone d'origine maternelle joue un rôle dans le contrôle des dépôts cuticulaires, du moins des plus précoces, du développement embryonnaire ; les recherches s'orientent vers la signification physiologique des conjugués d'ecdysone transférés de la mère à l'embryon (sont-ils hydrolysés lors de la production des pics d'ecdysone ?) ainsi que vers le rôle joué dans l'œuf par les déoxyecdysones d'origine maternelle (rôle de précurseurs de la biosynthèse ? rôle hormonal propre ?).

Présenté au Colloque D. G. R. S. T. de Port Boil, 27 février-1 er mars 1979. Accepté en octobre 1979.

Remerciements. - Les recherches faites dans le laboratoire de l'auteur ont bénéficié du soutien financier généreux de la DGRST (AC Biologie de la Reproduction et du Développement 75.7.1639 et 77.7.0656). Les résultats ont été obtenus en collaboration avec M. Lagueux, F. Goltzené, M. Charlet, C. Hetru et C. Jacquot qui travaillent dans le 
groupe de l'auteur, ainsi que M. Hirn du Centre d'Immunologie de Marseille-Luminy et Luu Bang du Laboratoire de Chimie Organique des Produits Naturels de l'Université Louis Pasteur de Strasbourg.

\section{Références}

BOLLENBACHER W. E., ZVENKO H., KUMARAN A. K., GILBERT L. I., 1978. Changes in ecdysone content during post-embryonic development of the wax moth, Galleria mellanella : the role of the ovary. Gen. comp. Endocr., 34, 169-179.

BORDEREAU C., HIRN M., DELBECQUE J. P., DE REGGI M., 1976. Présence d'ecdysones chez un insecte adulte : la reine de Termite. C. R. Acad. Sci., Paris (D), 282, 885-888.

BOROVSKY D., VAN HANDEL E., 1979. Does ovarian ecdysone stimulate Mosquitoes to synthesize vitellogenin ? J. Insect Physiol, 25, 861-866.

BUTENANDT A., KARLSON P., 1954. Uber dies Isolierung eines Metamorphosehormons der Insekten in kristallisierter Form. Zeitschr. Naturforsch., B9, 389-391.

DELBECQUE J. P., LANZREIN B., BORDEREAU C., IMBODEN H., HIRN M., O'CONNOR J. D., NOIROT C., LUSCHER M., 1978. Ecdysone and ecdysterone in physogastric Termite queens and eggs of Macrotermes bellicosus and Macrotermes subhyalinus. Gen. Comp. Endocr., 36, 40-47.

DE REGGIM. L., HIRN M. H., DELAAGE M. A., 1975. Radioimmunoassay of ecdysone. An application to Drosophila larvae and pupae. Biochem. biophys. Res. comm., 66, 1307-1315.

FALLON A. M., HAGEDORN H. H., WYATT G. R., LAUFER H., 1974. Activation of vitellogenin synthesis in the Mosquito Aedes aegypti by ecdysone. J. Insect Physiol., 20, 1815-1823.

GANDE A. R., MORGAN E. D., 1979. Ecdysteroids in the developing eggs of the desert locust, Schistocerca gregaria. J. Insect Physiol., 25, 289-294.

GOLTZENÉ F., 1977. Contribution à l'étude de l'ovogenèse chez Locusta migratoria. Th., Univ. LouisPasteur, Strasbourg.

GOLTZENÉ F., LAGUEUX M., CHARLET M., HOFFMANN J. A., 1978. The follicle cell epithelium of maturing ovaries of Locusta migratoria : A new biosynthetic tissue for ecdysone. HoppeSeyler's Z. Physiol. Chem., 359, 1427-1434.

HAGEDORN H. H., 1974. The control of vitellogenesis in the Mosquito Aedes aegypti. Amer. Zool., 14, $1207-1217$.

HAGEDORN H. H., O'CONNOR J. D., FUCHS M. S., SAGE B., SCHLAEGER D. A., BOHM M. K., 1975. The ovary as a source of $\alpha$-ecdysone in an adult mosquito. Proc. Nat. Acad. Sci. USA, 72, 3255-3259.

HAGET A., 1953. Analyse expérimentale des facteurs de la morphogenèse embryonnaire chez le Coléoptère Leptinotarsa. Bull. Biol. Fr. et Belg., 87, 123-217.

HETRU C., LAGUEUX M., BANG LUU, HOFFMANN J. A., 1978. Adult ovaries of Locusta migratoria contain the sequence of biosynthetic intermediaies for ecdysone. Life Sciences, 22, 2141-2154.

HOFFMANN J. A., KOOLMAN J., BEYLER C., 1975. Rôle des glandes prothoraciques dans la production d'ecdysone au cours du dernier stade larvaire de Lacusta migratoria L. C. R. Acad. Sci., Paris (D), 280, 733-736.

HSIAO T. H., HSIAO C., 1979. Ecdysteroids in the ovary and the egg of the greater wax moth. J. Insect Physiol., 25, 45-52.

HUBER R., HOPPE W., 1965. Zur Chemie des Ecdysons. VIl. Die Kristall- und Molekül-Strukturanalyse des Insektenverpuppungshormons Ecdyson mit der automatisierten Faltmolekülmethode. Chem. Ber., 98, 2403-2423.

JACQUOT C., 1979. Rapport Stage DEA, UER Sciences Vie et Terre, Univ. Louis-Pasteur, Strasbourg. KARLSON P., HOFFMEISTER H., HOPPE W., HUBER R., 1963. Justus Liebigs Ann. Chem., 662, 1-20.

KARLSON P., STAMM-MENENDEZ D., 1956. Notiz über den Nachweis von Metamorphosehormon in den Imagines von Bombyx mori. Hoppe-Seyler's Z. Physiol. Chem., 306, 109-111.

LAGUEUX M., HIRN M., DE REGGI M. HOFFMANN J. A., 1976. Rôle de l'ecdysone au cours du développement ovarien de Locusta migratoria. C. R. Acad. Sci., Paris (D), 283, 1081-1084.

LAGUEUX M., HIRN M., HOFFMANN J. A., 1977. Ecdysone during ovarian development in Locusta migratoria. J. Insect Physiol., 23, 109-119. 
LEGAY J. M., CALVEZ B., HIRN M., DE REGGI M. L., 1976. Ecdysone and oocyte morphogenesis in Bombyx mori. Nature, Lond., 262, 489-490.

LUU BANG, LAGUEUX M., HIRN M., HOFFMANN J. A., 1976. Identification par chromatographie en phase vapeur et spectrométrie de masse de l'ecdysone synthétisée dans les ovaires de femelles adultes de Locusta migratoria (Insecte Orthoptère). C. R. Acad. Sci., Paris (D), 283, 1081-1084.

MÜLLER N. S., 1963. An experimental analysis of molting in embryos of Melanoplus differentialis. Dev. Biol., 8, 222-240.

OHNISHI E., MIZUNO T., IKEKAWA N., SAKURAI S., 1977o. 2-Deoxy- $\alpha$-ecdysone from ovaries and eggs of the silkworm, Bombyx mori. Science, Wash., 197, 66-67.

OHNISHI E., MIZUNO T., IKEKAWA N., AWATA N., SAKURAI S., 1977b. Occurrence of $\alpha$-ecdysone in the developing embryos of the silk-worm, Bombyx mori. J. Insect Physiol., 23, 317-319.

ONO S. E., NAGAYAMA H., SHIMURA K., 1975. The occurrence and synthesis of female and eggspecific proteins in the silkworm Bombyx mori. Insect Biochem., 5, 313-330.

SBRENNA-MICCIARELLI A., SBRENNA G., 1972. The embryonic apolyses of Schisfocerca gregaria (Orthoptera). J. Insect Physiol., 18, 1027-1037.

SCHLAEGER D. A., FUCHS M. S., KANG S. H., 1974. Ecdysone mediated stimulation of Dopadecarboxylase activity and its relation to ovarian development in Aedes aegrpti. J. Cell Biol., 61, 454-465. 